\title{
ILC for FES-based Stroke Rehabilitation of Hand and Wrist
}

\author{
Anna Soska, Chris Freeman and Eric Rogers
}

\begin{abstract}
Functional electrical stimulation (FES) has been shown to be an effective technique for stroke rehabilitation, but when applied to support wrist and hand movement, current controllers in clinical use are almost exclusively open-loop which degrades their accuracy and hence therapeutic potential. As one of the few model-based control approaches to be used in clinical trials for the upper limb, iterative learning control (ILC) has clear potential in this area. This paper develops a clinicallyrelevant model of the hand and wrist incorporating stimulated muscles to assist the implementation of FES based rehabilitation system and establishes the ability of ILC to provide precision motion control over repeated attempts at a task. Practical considerations are discussed relating to the application of the control algorithms developed within stroke rehabilitation.
\end{abstract}

\section{INTRODUCTION}

Stroke is the leading cause of serious, long-term disability worldwide and typically results in partial paralysis on one side of the body, termed hemiplegia. Many patients lose the ability to use their hand, often because of a loss of dexterity, weakness in opening the hand due to finger extension deficit and, over time, increasing stiffness due to spasticity and muscle contractures. Fibers killed in a cerebrovascular accident cannot re-grow, but through intensive and systematic task repetition the brain is able to form new connections, with redundant ones disappearing. Conventional therapy is of limited benefit in improving impaired upper limb function and, driven by an aging population and increasing costs, there is a pressing need to improve the effectiveness of treatment, as well as shift the responsibility for good health from health professionals to patients.

A body of clinical evidence exists to support functional electrical stimulation (FES) as a promising technology to assist stroke patients in regaining motor function [1] and it is an increasingly active area within rehabilitation engineering [2], [3] with theoretical support from neurophysiology and motor learning research. The therapeutic effect of FES has been shown to be enhanced when it is associated with patient's voluntary movement [4], motivating precise FEScontrollers capable of accurately assisting repeated tasks, whilst also promoting the patient's voluntary contribution to the movement. Unfortunately very few model-based control approaches capable of accurately assisting movement have been employed clinically [5], [6], an environment with minimal set-up time, reduced control over environmental constraints and little possibility of repeating any one test in the program of treatment undertaken, controllers are required to perform to a minimum standard on a wide number of

The authors are with the School of Electronics and Computer Science, University of Southampton, SO17 1DP, UK. ais106@ecs.soton.ac.uk. subjects and conditions. In particular, for the hand and wrist only open-loop approaches have been used clinically for rehabilitation and hence low accuracy has been achieved which limits the potential therapeutic effect of treatment.

Iterative learning control (ILC) is one model-based approach that has been applied in two clinical trials with stroke patients, and exploits the repetitive nature of the task. ILC is suitable for any process which repeats the same finite duration task over and over again, and is a very active area of research, see, for example, [7]. In the first clinical trial FES was applied to the triceps muscle, and ILC updated the stimulation level to assist patients' completion of a planar reaching task. In particular, each patient's arm was strapped to a robot and they attempted to follow a target moving along an illuminated elliptical track defined over the time duration. At the end of this period, the robot returned their arm to the starting position, and in this resetting time an ILC algorithm was used to update the stimulation to be applied on the next attempt [8]. The ability of ILC to modify the input in response to physiological changes which would otherwise erode performance gave rise to highly accurate tracking. During the course of the clinical trial, each patient's voluntary effort increased and the level of FES required reduced [9], with outcome measures confirming significant increase in arm function following treatment. This approach has recently been extended by applying FES to two muscles in the arm and shoulder [10] to assist 3D movements of the arm. This again yielded significant improvement in unassisted movement capability when applied during a clinical trial with stroke patients. Hand and wrist function, however, is a critical component of activities of daily living, and hence neglecting to train them severely reduced the potential of both systems. This is also a common feature of another emerging technology, rehabilitation robotics, where the wrist and hand is often omitted [11] due to its inherent complexity.

This paper establishes the feasibility of employing ILC to provide accurate movement control of the hand and wrist for stroke rehabilitation using FES. To do this a suitable model is constructed and an ILC scheme is developed to achieve tracking in the presence of practical constraints on the system. Performance when using only extrinsic muscles, that are compatible with using surface FES, is compared against using both extrinsic and intrinsic muscles. Simulation studies confirm feasibility and establish efficacy for clinical use to extend the potential of FES-based rehabilitation.

\section{FES CONTROL STRUCTURE}

The FES control scheme is required to generate suitable electrical pulse signals to activate appropriate muscles in the 
hand and wrist, these developing muscle forces that combine to produce a desired movement of the musculoskeletal system. The current/voltage amplitude or the width of the stimulus pulses may be the controlled variable, although the latter is preferable since it provides a more consistent response across subjects, requires a smaller charge per stimulus pulse, and allows for greater selectivity of recruitment. The proposed control scheme is shown in Fig. 1.

The system considered is highly coupled with each joint actuated by at least two muscle groups (flexor and extensors). Furthermore most of the hand muscles are either bi-articular or multi-articular, which means they actuate simultaneously two or more joints.

The muscles of the hand are divided into intrinsic muscles, which originate solely in the hand and extrinsic muscles located proximally in the forearm. Although it is possible to stimulate individual intrinsic and extrinsic muscles of the hand i.e. using embedded electrodes, in most of the FES systems only the extrinsic muscles are stimulated. The approach taken in this paper is to investigate feasibility of surface electrode array stimulation of extrinsic muscles, which hence avoids the need for surgery, making the approach cost effective and suitable for widespread uptake.

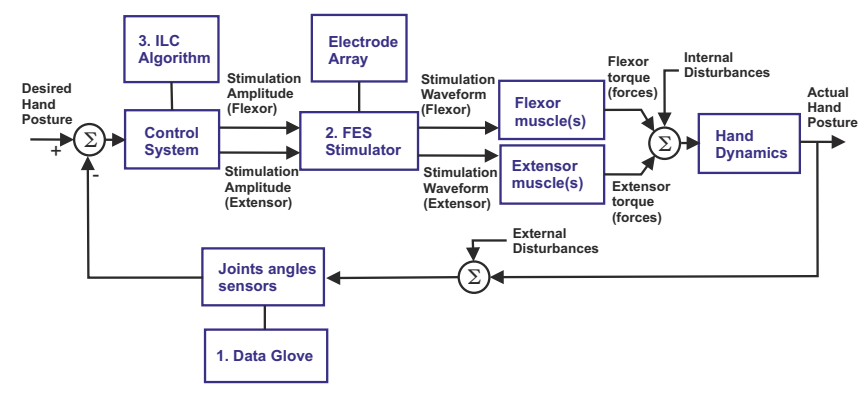

Fig. 1. FES schematic for hand and wrist rehabilitation.

\section{MODEL OF THE WRIST AND HAND}

In this work a simplified model suitable for clinical use is developed in order to confirm control performance. The model includes a single composite finger, representing the combined action of four fingers and wrist and neglects the thumb orientation. The finger and wrist are modeled as a 3link rigid body system, consisting of 3 active revolute joints, as shown in Fig. 2. This still provides an accurate representation of the hand since $42 \%$ of the functional movements of the hand involve the four fingers moving together [12]. Link 1 represents the II-V Metacarpal bones connected by the wrist joint, Links 2 and 3 represent proximal and middle phalangeals of the finger connected by the MetacarpalPhalangeal joint (MCP) and Proximal-Interphalangeal joint (PIP) respectively.

The dynamic model of the finger/wrist is formulated using the Lagrange method and can be rewritten in the form

$$
\mathbf{M}(\mathbf{q}) \ddot{\mathbf{q}}+\mathbf{C}(\mathbf{q}, \dot{\mathbf{q}})+\mathbf{G}(\mathbf{q})+\mathbf{F}(\mathbf{q}, \dot{\mathbf{q}})=\boldsymbol{\tau}
$$

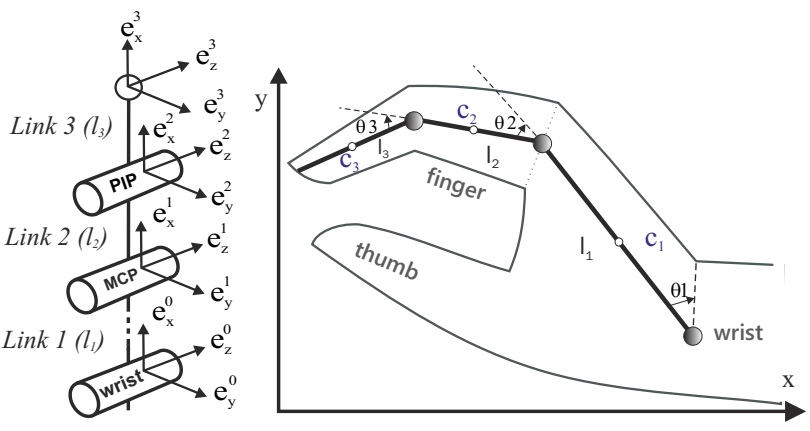

Fig. 2. Planar hand model

where $\boldsymbol{M}(\boldsymbol{q})$ is the inertia matrix, $\boldsymbol{C}(\boldsymbol{q}, \dot{\boldsymbol{q}})$ denotes the centrifugal and Coriolis forces, $\boldsymbol{G}(\boldsymbol{q})$ is the vector of gravitational force, and the generalized coordinates of the system are $\mathbf{q}=\left[\begin{array}{lll}\theta_{1}, & \theta_{2}, & \theta_{3}\end{array}\right]^{T}$. During purely horizontal movement of the finger, gravity can be neglected.

The elements of the symmetric inertia matrix are

$$
\begin{aligned}
m_{11} & =m_{1} c_{1}^{2}+m_{2} l_{1}^{2}+m_{2} c_{2}^{2}+2 m_{2} l_{1} c_{2} \cos \theta_{2}+m_{3} l_{1}^{2} \\
& +m_{3} l_{2}^{2}+2 m_{3} l_{1} l_{2} \cos \theta_{2}+2 m_{3} l_{1} c_{3} \cos \left(\theta_{2}+\theta_{3}\right) \\
& +2 m_{3} l_{2} c_{3} \cos \theta_{3}+m_{3} c_{3}^{2}+J_{1}+J_{2}+J_{3} \\
m_{12} & =m_{2}\left(c_{2}^{2}+l_{1} c_{2} \cos \theta_{2}\right)+m_{3} l_{2}^{2}+m_{3} c_{3}^{2}+m_{3} l_{1} l_{2} \cos \theta_{2} \\
& +m_{3} l_{1} c_{3} \cos \left(\theta_{2}+\theta_{3}\right)+2 m_{3} l_{2} c_{3} \cos \theta_{3}+J_{2}+J_{3} \\
m_{13} & =m_{3} c_{3}^{2}+m_{3} l_{1} c_{3} \cos \left(\theta_{2}+\theta_{3}\right)+m_{3} l_{2} c_{3} \cos \theta_{3}+J_{3}, \\
m_{22} & =m_{2} c_{2}^{2}+m_{3} l_{2}^{2}+m_{3} c_{3}^{2}+m_{3} l_{2} c_{3} \cos \theta_{3}+J_{3}, \\
m_{23} & =m_{3} c_{3}^{2}+m_{3} l_{2} c_{3} \cos \theta_{3}+J_{3} \\
m_{33} & =m_{3} c_{3}^{2}+J_{3}
\end{aligned}
$$

where $m_{1}=0.3, m_{2}=0.015, m_{3}=0.009$ are masses in $[k g], J_{1}=5 e-4, J_{2}=5 e-6, J_{3}=3 e-6$, are inertias in $\left[\mathrm{kg} \cdot \mathrm{m}^{2}\right]$, and the assumed lengths in $[\mathrm{m}]$ are $l_{1}=0.08$, $l_{2}=0.05, l_{3}=0.048$ and $c_{i}=0.5 l_{i}, i=1,2,3$. The elements of $\mathbf{C}(\mathbf{q}, \dot{\mathbf{q}})$ are

$$
\begin{aligned}
c_{11}= & -\left[m_{3} c_{3} l_{1} s_{23}+m_{3} c_{3} l_{2} \sin \theta_{3}\right]\left(2 \dot{\theta_{1}} \dot{\theta_{3}}+2 \dot{\theta_{2}} \dot{\theta_{3}}+\dot{\theta_{3}^{2}}\right) \\
- & {\left[\left(m_{2} l_{1} c_{2}+m_{3} l_{1} l_{2}\right) \sin \theta_{2}+m_{3} l_{1} c_{3} s_{12}\right]\left(2 \dot{\theta_{1}} \dot{\theta_{2}}+\dot{\theta_{2}^{2}}\right) } \\
c_{21}= & {\left.\left[m_{2} c_{2} l_{1}+m_{3} l_{1} l_{2}\right) \sin \theta_{2}+m_{3} c_{3} l_{2} s_{23}\right] \dot{\theta_{1}^{2}} } \\
& -m_{3} c_{3} l_{2} \sin \theta_{3}\left(2 \dot{\theta_{1}} \dot{\theta_{3}}+2 \dot{\theta_{2}} \dot{\theta_{3}}+\dot{\theta_{3}^{2}}\right) \\
c_{31}= & {\left[m_{3} c_{3} l_{2} \sin \theta_{3}+m_{3} c_{3} l_{1} s_{23}\right] \dot{\theta_{1}^{2}} } \\
& +m_{3} c_{3} l_{2} \sin \left(2 \dot{\theta_{1}} \dot{\theta_{2}}+\dot{\theta_{2}^{2}}\right)
\end{aligned}
$$

where $s_{i j}=\sin \left(\theta_{i}+\theta_{j}\right)$. The vector of moments produced through application of FES is $\boldsymbol{\tau}$, and $\boldsymbol{F}(\boldsymbol{q}, \dot{\boldsymbol{q}})$ is the vector of frictional components acting about each joint, with the form

$$
\boldsymbol{F}(\boldsymbol{q}, \dot{\boldsymbol{q}})=\left[\begin{array}{c}
k_{1}\left(\theta_{0,1}-\theta_{1}\right)-b_{1} \dot{\theta}_{1} \\
k_{2}\left(\theta_{0,2}-\theta_{2}\right)-b_{2} \dot{\theta}_{2} \\
k_{3}\left(\theta_{0,3}-\theta_{3}\right)-b_{3} \dot{\theta}_{3}
\end{array}\right]
$$

where $b_{1}=0.05, b_{2}=0.014, b_{3}=0.01$ are the damping coefficients in $[N \cdot s / m]$. It has been assumed that the muscle groups which actuate each joint produce a stiffness that may 
be represented by a spring with zero elongation at the initial position $\theta_{0,1}=\frac{2}{3} \pi, \theta_{0,2}=\frac{\pi}{2}, \theta_{0,3}=\frac{\pi}{3}$ in [rad] and with stiffness coefficients $k_{1}=6.5, k_{2}=0.9, k_{3}=0.8$ in $[\mathrm{N} / \mathrm{m}]$.

The musculoskeletal structure of the finger and wrist is shown in Fig. 3. The wrist joint is assumed to be actuated by three extensor muscles: Extensor Communis (EC), Extensor Carpi Radialis Longus (ECR) and Extensor Carpi Ulnaris (ECU). The muscles of the finger act through a complex tendon network, termed the extensor mechanism. The network is approximated by a longitudinally symmetric tendon rhombus, consisting of active and the passive tendons, as shown in Fig. 3. The extensor mechanism of the finger is

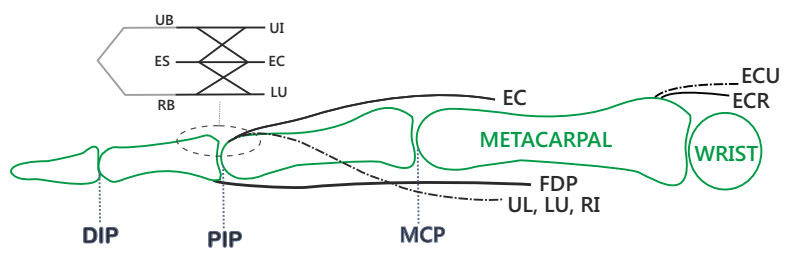

Fig. 3. The musculo-tendon structure of the wrist and finger

modeled as in [13] and includes 5 active tendons, driven by independently controlled muscles: the Flexor Digitorum Profundus (FDP), the Extensor Digitorum Communis (EC), the Ulnar and Radial Interosseous (UI and RI), the Lumbrical muscle (LU), and 3 passive tendons: the Radial Band (RB) the Ulnar Band (UB) and the Extensor Slip (ES).

Electrically stimulated muscles of the hand contract, generating pulling forces that produce finger/wrist movement. The transformation from muscle force vector, $\boldsymbol{y}$, with dimension $m$ to the $p$-dimensional net joint torque, $\boldsymbol{\tau}$, at the finger/wrist joints can be defined as in [14], yielding

$$
\boldsymbol{\tau}=\underbrace{\left[\begin{array}{ccc}
r_{11} & \ldots & r_{1 m} \\
\vdots & \ddots & \vdots \\
r_{p 1} & \ldots & r_{p m}
\end{array}\right]}_{\mathbf{R}(\mathbf{q})} \underbrace{\left[\begin{array}{c}
y_{1}\left(u_{1}, l_{1}, i_{1}\right) \\
\vdots \\
y_{m}\left(u_{m}, l_{m}, i_{m}\right)
\end{array}\right]}_{\mathbf{y}(\mathbf{u}, \mathbf{q}, \dot{\mathbf{q}})}
$$

where $\mathbf{R}(\mathbf{q})$ is a matrix of moment arms in which each entry is the signed scalar moment arm value that transforms a muscle force into torques at the various joints it crosses.

The $r_{i j}$ element of the moment arm matrix can be evaluated by differentiating the excursion (displacement) $E$ of the $j^{\text {th }}$ tendon with respect to the $i^{t h}$ joint angle

$$
r_{i j}=\frac{\partial E_{j}\left(\theta_{i}\right)}{\partial \theta_{i}}, \quad i=1, \ldots p, \quad j=1, \ldots m .
$$

The excursion of the FDP is modeled using the III Landsmeer's model [15]. This gives the following expression for excursion

$$
E^{\text {tendon }}=\theta d^{\text {tendon }}+2 y^{\text {tendon }}\left(1-\frac{\theta / 2}{\tan (\theta / 2)}\right)
$$

where $d^{t e n d o n}$ is the distance from the straight section of the tendon to the tendon constraint along the line perpendicular to the axis of the bone and $\theta$ is the corresponding angle rotation. The term $y^{\text {tendon }}$ is the distance along the axis of the bone from the end of the straight section of the tendon to the joint centre.

The tendon excursion of the EC is a function of the wrist and MCP with the addition of the displacement, transformed to the PIP joint through the extensor mechanism.

$$
E^{E C}=-r_{1}^{E C} \theta_{1}-r_{2}^{E C} \theta_{2}+L\left(E_{1}, E_{2}, E_{3}\right)
$$

where

$$
E_{1}=E^{E S}, \quad E_{2}=E^{U B}, \quad E_{3}=E^{R B}
$$

and $L\left(E_{1}, E_{2}, E_{3}\right)$ is the excursion function defined as

$$
L\left(E_{1}, E_{2}, E_{3}\right)=\sum_{i=1}^{3} w_{i} E_{i}=0, \quad w_{i}>0 \quad \forall j=1,2,3
$$

The excursions of the remaining tendons are each modeled as a second order polynomial approximation of (7)

$$
\left(b^{\text {tendon }}+h_{\text {tendon }} \theta\right) \theta
$$

where $b^{\text {tendon }}$ and $h^{\text {tendon }}$ are constants.

The equations of tendon excursions in the considered case can therefore be expressed as functions of finger extension/flexion angles $\theta_{2}$ and $\theta_{3}$ as follows

$$
\begin{aligned}
E^{F D P} & =\sum_{i=2}^{3} \theta_{i}^{F D P}+2 y_{i}^{F D P}\left(1-\frac{\theta_{i} / 2}{\tan \left(\theta_{i} / 2\right)}\right) \\
E^{E S} & =-r^{E S} \theta_{3} \\
E_{R B} & =-\left(b^{R B}+h^{R B} \theta_{3}\right) \theta_{3} \\
E_{U B} & =-\left(b^{U B}+h^{U B} \theta_{3}\right) \theta_{3} \\
E^{R I} & =\left(b^{R I}+h^{R I} \theta_{2}\right) \theta_{2}+E^{U B} \\
E^{U I} & =\left(b^{U I}+h^{U I} \theta_{2}\right) \theta_{2}+E^{U B} \\
E^{L U} & =\left(b^{L U}+h^{L U} \theta_{2}\right) \theta_{2}+E^{R B}-E^{F D P} \\
E^{E C U} & =\left(b^{E C U}+h^{E C U} \theta_{1}\right) \theta_{1} \\
E^{E C R} & =\left(b^{E C R}+h^{E C R} \theta_{1}\right) \theta_{1}
\end{aligned}
$$

Hence, applying (6), each column of moment arm matrix $\mathbf{R}(\mathbf{q})$ represents the moment arm vector corresponding to each muscle, yielding

$$
\mathbf{R}(\mathbf{q})=\left[\mathbf{R}^{F D P}, \mathbf{R}^{L U}, \mathbf{R}^{U I}, \mathbf{R}^{R I}, \mathbf{R}^{E C}, \mathbf{R}^{E C R}, \mathbf{R}^{E C U}\right]
$$

Here the moment arm vector for FDP equals

$$
\mathbf{R}^{F D P}=\left[\begin{array}{c}
0 \\
d_{1}^{F D P}+y_{1}^{F D P}\left(\frac{\sin \left(\theta_{2}\right)-\theta_{2}}{2 \sin ^{2}\left(\theta_{2}\right)}\right) \\
d_{2}^{F D P}+y_{2}^{F D P}\left(\frac{\sin _{3}\left(\theta_{3}\right)-\theta_{3}}{2 \sin ^{2}\left(\theta_{3}\right)}\right)
\end{array}\right]
$$

and the moment arm vector for $\mathrm{LU}$ is given by

$$
\mathbf{R}^{L U}=\left[\begin{array}{c}
0 \\
b^{L U}+2 h^{L U} \theta_{2}-R_{\theta_{2}}^{F D} \\
-b^{R B}-2 h^{R B} \theta_{3}-R_{\theta_{3}}^{F D P}
\end{array}\right]
$$

The moment arm vector for UI equals

$$
\mathbf{R}^{U I}=\left[\begin{array}{c}
0 \\
b^{U I}+2 h^{U I} \theta_{2} \\
-b^{U B}-2 h^{U B} \theta_{3}
\end{array}\right]
$$


and the moment arm vector for EC has the form

$$
\mathbf{R}^{E C}=\left[\begin{array}{c}
-r_{1}^{E C} \\
-r_{2}^{E C} \\
-w_{1} r^{E S}+w_{2} R_{\theta_{3}}^{U B}+w_{3} R_{\theta_{3}}^{R B}
\end{array}\right]
$$

where

$$
\begin{aligned}
& R_{\theta_{3}}^{U B}=-\left(b^{U B}+2 h^{U B} \theta_{3}\right) \\
& R_{\theta_{3}}^{R B}=-\left(b^{R B}+2 h^{R B} \theta_{3}\right)
\end{aligned}
$$

The moment arm vector for ECR equals

$$
\boldsymbol{R}^{E C R}=\left[b^{E C R}+2 h^{E C R}, \quad 0, \quad 0\right]^{T}
$$

and the moment arm vectors for ECU is

$$
\boldsymbol{R}^{E C U}=\left[b^{E C U}+2 h^{E C U}, \quad 0, \quad 0\right]^{T}
$$

Using relation (5), the torque acting about joint can now be calculated as a function of the force in each muscle and the current joint angle vector.

\begin{tabular}{|l|c|c|c|c|c|}
\hline Tendon - type (joint) & $\mathrm{d}$ & $\mathrm{y}$ & $\mathrm{r}$ & $\mathrm{h}$ & $\mathrm{b}$ \\
\hline EC - extrinsic (wrist) & - & - & 14.12 & - & - \\
\hline ECR - extrinsic (wrist) & - & - & - & -11.72 & 1.14 \\
\hline ECU - extrinsic (wrist) & - & - & - & -8.51 & 1.55 \\
\hline FDP - extrinsic (MCP) & 8.32 & 8.32 & - & - & - \\
\hline RI - intrinsic (MCP) & - & - & - & -1.29 & 5.62 \\
\hline UI - intrinsic (MCP) & - & - & - & -8.16 & 18.76 \\
\hline LU - intrinsic (MCP) & - & - & - & -2.17 & 12.53 \\
\hline EC (MCP) & - & - & 8.3 & - & - \\
\hline FDP (PIP) & 5.76 & 7.5 & - & - & - \\
\hline ES - intrinsic (PIP) & - & - & 2.92 & - & - \\
\hline RB - intrinsic (PIP) & - & - & - & -0.47 & 2.54 \\
\hline UB - intrinsic (PIP) & - & - & - & 0.57 & 1.7 \\
\hline
\end{tabular}

Each element of the muscle force vector $\mathbf{y}(\mathbf{u}, \mathbf{q}, \dot{\mathbf{q}})$ comprises the moment produced through the application of FES signal $u_{j}(t)$ to the $j^{t h}$ stimulated muscle, with

$$
\boldsymbol{u}=\left[\begin{array}{lll}
u_{1} & \cdots & u_{m}
\end{array}\right]^{T}
$$

As discussed in [16], the most prevalent form of muscle representation is a Hill-type model of the form

$$
y_{i}\left(u_{i}(t), \mathbf{q}, \dot{\mathbf{q}}\right)=h_{i}\left(u_{i}, t\right) F_{m, i}\left(l_{m, i}, i_{m, i}\right), \quad i=1, \cdots, m
$$

Here the term, $h_{i}\left(u_{i}, t\right)$ is a Hammerstein structure incorporating a static non-linearity, $h_{I R C, i}\left(u_{i}\right)$, representing the isometric recruitment curve, cascaded with linear activation dynamics, $h_{L A D, i}(t)$. These typically are second order, and in the considered case are modeled as a second order critically damped system with a natural frequency $\omega_{n}=2.7$, and their state-space model matrices are denoted by $\mathbf{M}_{A, i}, \mathbf{M}_{B, i}$, $\mathbf{M}_{C, i}$. The term $F_{m, i}\left(l_{m, i}, \dot{l}_{m, i}\right)$ models the multiplicative effect of the muscle length $l_{m, i}$ and muscle velocity on the active torque developed by the muscle.

\section{NEWTON METHOD-BASED ILC}

As discussed, ILC is a methodology for improving tracking performance of systems that execute the same task repeatedly by learning from past actions. ILC is currently a very active research area, and has been implemented in many application fields [7]. In this section ILC is applied to the hand and wrist system in simulation to investigate feasibility and performance capabilities prior to experimental use.

\section{A. System description}

From (1) the relationship between stimulation and joint angles can be expressed in state-space form as

$$
\begin{aligned}
& \dot{\mathbf{x}}(t)=\left[\begin{array}{c}
\dot{\mathbf{q}} \\
\mathbf{M}(\mathbf{q})^{-1} \mathbf{X}(\mathbf{q}, \dot{\mathbf{q}}) \\
\mathbf{M}_{A, 1} x_{1} \\
\vdots \\
\mathbf{M}_{A, p} x_{p}
\end{array}\right]+\left[\begin{array}{c}
\mathbf{0} \\
\mathbf{0} \\
\mathbf{M}_{B, 1} h_{\mathrm{IRC}, 1}\left(u_{1}\right) \\
\vdots \\
\mathbf{M}_{B, p} h_{\mathrm{IRC}, p}\left(u_{p}\right)
\end{array}\right] \\
& :=\mathbf{f}(\mathbf{x}(t), \mathbf{u}(t)) \\
& \mathbf{q}(t)=\left[\begin{array}{llll}
\mathbf{I} & \mathbf{0} & \cdots & \mathbf{0}
\end{array}\right] \mathbf{x}(t):=\mathbf{h}(\mathbf{x}(t))
\end{aligned}
$$

where $\mathbf{x}=\left[\mathbf{q}^{T}, \dot{\mathbf{q}}^{T}, x_{1}^{T} \cdots x_{j}^{T}\right]^{T}$ and $\mathbf{X}(\mathbf{q}, \dot{\mathbf{q}})$ has $i^{\text {th }}$ row

$$
\mathbf{R}_{i}(\mathbf{q}) \mathbf{M}_{C, i} x_{i} F_{m, i}(\mathbf{q}, \dot{\mathbf{q}})-\mathbf{C}_{i}(\mathbf{q}, \dot{\mathbf{q}})-\mathbf{F}_{i}(\mathbf{q}, \dot{\mathbf{q}})
$$

Now discretise the nonlinear stimulated arm system (32) to produce

$$
\begin{aligned}
\mathbf{x}_{k}(t+1) & =\mathbf{f}\left(\mathbf{x}_{k}(t), \boldsymbol{u}_{k}(t)\right) \\
\mathbf{q}_{k}(t) & =\mathbf{h}\left(\mathbf{x}_{k}(t)\right)
\end{aligned}
$$

where $k=1,2, \ldots$ is the trial number, $t \in[0,1,2, \ldots, N-1]$ is the sample number and $\mathbf{x}_{k}(t), \boldsymbol{u}_{k}(t)$ and $\mathbf{q}_{k}(t)$ are the state, input and output vectors respectively on the $k^{t h}$ trial. To replace (34) with a set of algebraic equations in $\mathbb{R}^{N}$, define the shifted input and output vectors as

$$
\begin{aligned}
& \boldsymbol{u}_{k}=\left[\boldsymbol{u}_{k}(0)^{T}, \boldsymbol{u}_{k}(1)^{T}, \ldots, \boldsymbol{u}_{k}(N-1)^{T}\right]^{T} \in \mathbb{R}^{m N} \\
& \mathbf{q}_{k}=\left[\mathbf{q}_{k}(1)^{T}, \mathbf{q}_{k}(2)^{T}, \ldots, \mathbf{q}_{k}(N)^{T}\right]^{T} \in \mathbb{R}^{p N}
\end{aligned}
$$

and the relationship between the input and output time-series can be expressed by the following algebraic functions

$$
\begin{aligned}
\mathbf{q}_{k}(1) & =\mathbf{h}\left(\mathbf{x}_{k}(1)\right)=\mathbf{h}\left(\boldsymbol{f}\left(\mathbf{x}_{k}(0), \boldsymbol{u}_{k}(0)\right)\right) \\
& =\boldsymbol{g}_{1}\left(\boldsymbol{x}_{k}(0), \boldsymbol{u}_{k}(0)\right) \\
\vdots & \\
\mathbf{q}_{k}(N) & =\boldsymbol{h}\left(\boldsymbol{x}_{k}(N)\right)=\boldsymbol{h}\left(\boldsymbol{f}\left(\boldsymbol{x}_{k}(N-1), \boldsymbol{u}_{k}(N-1)\right)\right) \\
& =\boldsymbol{g}_{N}\left(\boldsymbol{x}_{k}(0), \boldsymbol{u}_{k}(0), \boldsymbol{u}_{k}(1), \ldots, \boldsymbol{u}_{k}(N-1)\right)
\end{aligned}
$$

Assuming $\boldsymbol{x}_{k}(0)=0$, (32) can hence be represented as

$$
\mathbf{q}_{k}=\boldsymbol{g}\left(\boldsymbol{u}_{k}\right), \quad \boldsymbol{g}(\cdot)=\left[\boldsymbol{g}_{1}(\cdot)^{T}, \boldsymbol{g}_{2}(\cdot)^{T}, \ldots, \boldsymbol{g}_{N}(\cdot)^{T}\right]^{T}
$$

To control hand posture it is necessary to specify the joint positions at a fixed number, $M \leq N$, of sample instants given by $1 \leq n_{1}<n_{2}<\cdots<n_{M} \leq N$. Let the prescribed joint positions at these instants be

$$
\mathbf{q}^{*}=\left[\mathbf{q}^{*}(0)^{T}, \mathbf{q}^{*}(1)^{T}, \ldots, \mathbf{q}^{*}(M-1)^{T}\right]^{T} \in \mathbb{R}^{p M}
$$

ILC can be considered an iterative numerical solution to the problem of finding a control input which solves

$\min _{\mathbf{u}} J(\mathbf{u}) \quad$ subject to $\quad \Lambda \mathbf{u} \preceq \mathbf{b}, \quad J(\mathbf{u})=\left\|\mathbf{q}^{*}-\Phi \mathbf{g}(\mathbf{u})\right\|_{2}^{2}$

Here $J(\mathbf{u})$ is the point-to-point error norm, and the $p M \times p N$ matrix $\Phi$ has block-wise entries

$$
\Phi_{i, j}= \begin{cases}I_{p} & j=n_{i}, \quad i=1,2 \ldots M \\ 0_{p} & \text { otherwise }\end{cases}
$$


where $I_{p}$ and $0_{p}$ are the $p \times p$ identity and zero matrices respectively. Due to the requirement that each FES input is bounded, $u_{m} \leq u_{i} \leq u_{M}$, it is necessary to apply vector inequality constraints on the system input of the form $\Lambda \mathbf{u} \preceq$ $\mathbf{b}$, where $\Lambda=[-I, I]^{T}$ and $\mathbf{b}=\left[u_{m} \ldots u_{m}, u_{M} \ldots u_{M}\right]^{T}$.

Temporarily neglecting the constraint, the iterative solution via the Newton method is

$$
\begin{aligned}
\mathbf{u}_{k+1} & =\mathbf{u}_{k}-\nabla^{2} J\left(\mathbf{u}_{k}\right)^{-1} \nabla J\left(\mathbf{u}_{k}\right) \\
& =\mathbf{u}_{k}+\left(\Phi \mathbf{g}^{\prime}\left(\mathbf{u}_{k}\right)\right)^{\dagger}\left(\mathbf{q}^{*}-\Phi \mathbf{g}\left(\mathbf{u}_{k}\right)\right)
\end{aligned}
$$

where $\mathbf{g}^{\prime}\left(\mathbf{u}_{k}\right)=\frac{\delta \mathbf{g}\left(\mathbf{u}_{k}\right)}{\delta \mathbf{u}}$, and in the ILC framework $\mathbf{q}^{*}-$ $\Phi \mathbf{g}\left(\mathbf{u}_{k}\right)$ is replaced with the experimental point-to-point error $\mathbf{e}_{k}=\mathbf{q}^{*}-\Phi \mathbf{q}_{k}$. The descent direction term in (37) is the solution $\overline{\mathbf{u}}$ to

$$
\min _{\overline{\mathbf{u}}}\|\overline{\mathbf{u}}\|_{2}^{2} \quad \text { subject to } \quad \Phi \mathbf{g}^{\prime}\left(\mathbf{u}_{k}\right) \overline{\mathbf{u}}=\mathbf{e}_{k}
$$

and hence applying the constraint $\Lambda \mathbf{u}_{k+1} \preceq \mathbf{b}$, which translates to $\Lambda \overline{\mathbf{u}} \preceq \mathbf{b}-\Lambda \mathbf{u}_{k}$, means that (35) is solved using

$$
\mathbf{u}_{k+1}=\mathbf{u}_{k}+\Delta \mathbf{u}_{k}
$$

with $\Delta \mathbf{u}_{k}$ the solution to

$$
\min _{\overline{\mathbf{u}}}\|\overline{\mathbf{u}}\|_{2}^{2} \quad \text { subject to } \quad\left\{\begin{array}{rll}
\Phi \mathbf{g}^{\prime}\left(\mathbf{u}_{k}\right) \overline{\mathbf{u}} & =\mathbf{e}_{k} \\
\Lambda \overline{\mathbf{u}} & \preceq & \mathbf{b}-\Lambda \mathbf{u}_{k}
\end{array}\right.
$$

Using results in [17], this problem can be solved applying the gradient method to

$\min _{\mathbf{u}}\left\|\mathbf{y}_{r}-\Phi \mathbf{y}_{k}-\Phi \mathbf{g}^{\prime}\left(\mathbf{u}_{k}\right) \mathbf{u}\right\|_{2}^{2} \quad$ subject to $\Lambda \mathbf{u} \preceq \mathbf{b}-\Lambda \mathbf{u}_{k}$

using the barrier method, with corresponding update

$$
\mathbf{u}_{j+1}=\mathbf{u}_{j}+\alpha\left(\Phi \mathbf{g}^{\prime}\left(\mathbf{u}_{k}\right)\right)^{T}\left(\mathbf{e}_{k}-\Phi \mathbf{g}^{\prime}\left(\mathbf{u}_{k}\right) \mathbf{u}_{j}\right)-\frac{1}{\varsigma_{j}} \Lambda^{T} \mathbf{d}
$$

applied to the plant $\Phi \mathbf{g}^{\prime}\left(\mathbf{u}_{k}\right)$, where the elements of $\mathbf{d}$ are given by $d_{i}=1 /\left(b_{i}-\boldsymbol{\Lambda}_{i}^{T}\left(\mathbf{u}_{j}+\mathbf{u}_{k}\right)\right)$. This is performed multiple times between trial $k$ and $k+1$ in order to generate the decent term $\Delta \mathbf{u}_{k}$ used in (39). The parameter $\varsigma_{j}$ is increased each inter-trial update $j$ in order to reach the hard constraint, as described in [17]. Note that no computationally extensive matrix calculations are required within (40) since $\boldsymbol{w}=\mathbf{g}^{\prime}\left(\mathbf{u}_{k}\right) \boldsymbol{v}$ corresponds to the linear time-varying system

$$
\begin{aligned}
\tilde{\boldsymbol{x}}(t+1) & =\boldsymbol{A}(t) \tilde{\boldsymbol{x}}(t)+\boldsymbol{B}(t) \boldsymbol{v}(t) \\
\boldsymbol{w}(t) & =\boldsymbol{C}(t) \tilde{\boldsymbol{x}}(t)+\boldsymbol{D}(t) \boldsymbol{v}(t) \quad t=0, \ldots, N-1
\end{aligned}
$$

where

$$
\begin{array}{rlrl}
\boldsymbol{A}(t) & =\left(\frac{\partial \mathbf{f}}{\partial \boldsymbol{x}}\right)_{\mathbf{u}_{k}(t), \boldsymbol{x}_{k}(t)}, & \boldsymbol{B}(t) & =\left(\frac{\partial \mathbf{f}}{\partial \mathbf{u}}\right)_{\mathbf{u}_{k}(t), \mathbf{x}_{\mathbf{k}}(\mathbf{t})} \\
\boldsymbol{C}(t) & =\left(\frac{\partial \mathbf{h}}{\partial \boldsymbol{x}}\right)_{\mathbf{u}_{k}(t), \boldsymbol{x}_{k}(t)}, & \boldsymbol{D}(t)=\left(\frac{\partial \mathbf{h}}{\partial \boldsymbol{u}}\right)_{\mathbf{u}_{k}(t), \boldsymbol{x}_{k}(t)}
\end{array}
$$

Similarly the term $\boldsymbol{w}=\left(\boldsymbol{g}^{\prime}\left(\boldsymbol{u}_{k}\right)\right)^{T} \boldsymbol{v}$ equates to the system

$$
\begin{aligned}
\tilde{\boldsymbol{x}}(t+1) & =\boldsymbol{A}^{T}(t) \tilde{\boldsymbol{x}}(t)+\boldsymbol{C}^{T}(t) \boldsymbol{v}(N-1-t) \\
\boldsymbol{w}(N-1-t) & =\boldsymbol{B}^{T}(t) \tilde{\boldsymbol{x}}(t)+\boldsymbol{D}^{T}(t) \boldsymbol{v}(N-1-t)
\end{aligned}
$$

Convergence and robustness properties are given in [17], and in particular, convergence to zero error requires that
$\Phi \mathbf{g}^{\prime}\left(\mathbf{u}_{k}\right)$ has full row rank. This thereby allows point-to-point locations to be chosen to recover feasibility in the presence of a high coupled interaction matrix $\mathbf{R}(\mathbf{q})$.

\section{SIMULATION EVALUATION}

Simulation results are given using a sampling frequency of $100 \mathrm{~Hz}$. The clinically relevant task is to move the hand from initial flexed position to position $\theta_{1}=1.57 \mathrm{rad}$, $\theta_{2}=0.47 \mathrm{rad}$ and $\theta_{3}=0.21 \mathrm{rad}$, representing opening the hand to grasp an object. To investigate the feasibility of surface FES two separate cases are considered 1) FES is applied to all muscles, 2) FES is applied only to extrinsic muscles. Practical stimulation limits of $u_{m}=0, u_{M}=$ $350 \mu s$ are applied. Initial simulation results show that a wide variety of point-to-point movements can be achieved via extrinsic muscle stimulation, but this requires higher levels of stimulation. Figures 4 and 6 show joint trajectories over 10 trials, as well as final trial FES inputs. As seen in Fig. 5 and 7 , error convergence in both cases shows high accuracy achieved within few trials.
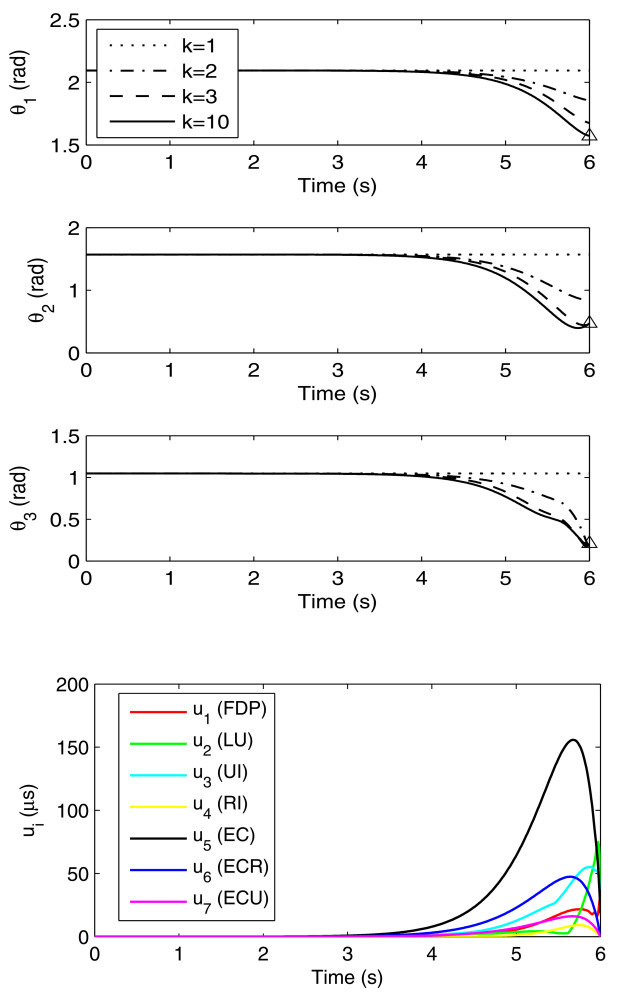

Fig. 4. Stimulation of extrinsic and intrinsic muscles using Newton methodbased point-to-point ILC with inequality constraint.

The results hence confirm the potential of assisting movement using those muscles that are compatible with surface array FES but indicate increased input norms, and hence likelihood of fatigue, especially for the EC muscle. 


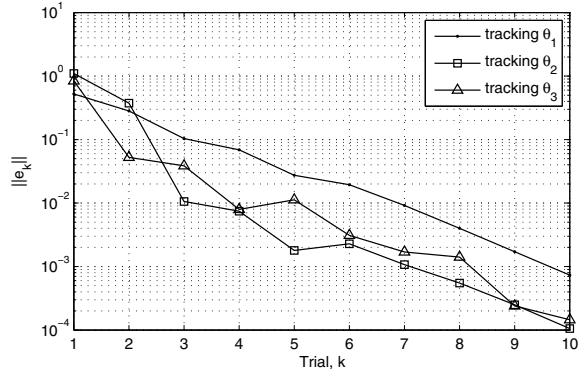

Fig. 5. Stimulation of extrinsic and intrinsic muscles using Newton methodbased point-to-point ILC: error norm.
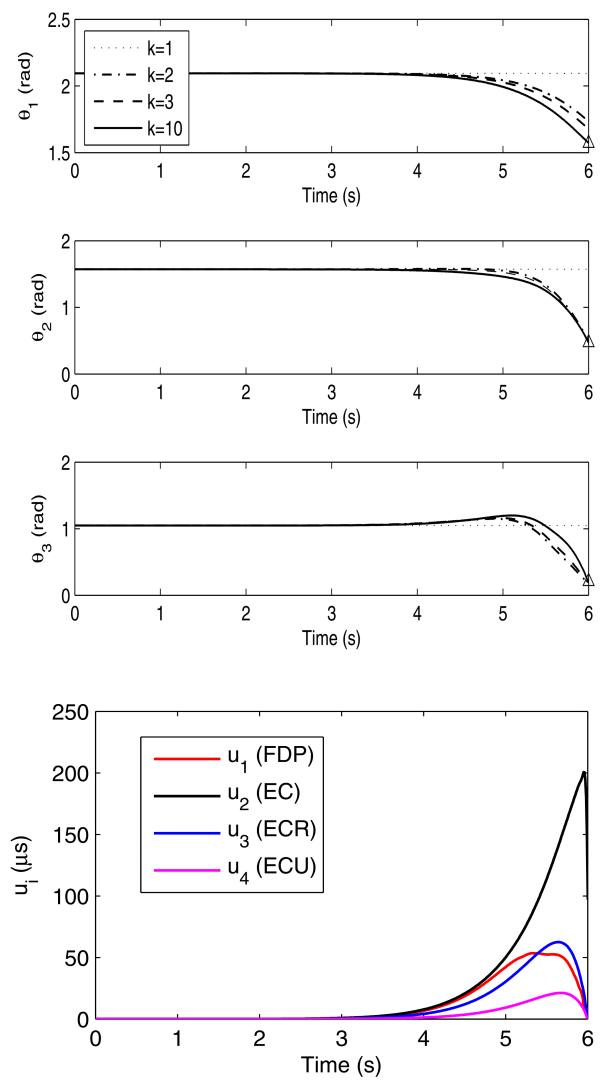

Fig. 6. Stimulation of extrinsic muscles using Newton method-based pointto-point ILC with inequality constraint.

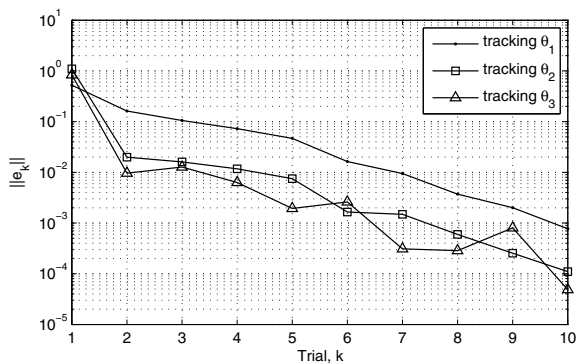

Fig. 7. Stimulation of extrinsic muscles using Newton method-based pointto-point ILC with inequality constraint: error norm.

\section{CONCLUSIONS AND FUTURE WORK}

Through development of a model of the hand and wrist suitable for hand rehabilitation, together with development of an ILC framework which addresses practical performance considerations, this paper has established the feasibility of model-based control of the hand and wrist using surface FES. The ILC algorithms developed will next be evaluated on both unimpaired as well as stroke participants before being used in clinical trials for the purpose of rehabilitation.

\section{REFERENCES}

[1] V. M. Pomeroy, L. M. King, A. Pollock, A. Baily-Hallam, and P. Langhorne, "Electrostimulation for promoting recovery of movement or functional ability after stroke: a review," Cochrane Database of Systematic Reviews, 2006.

[2] J. R. D. Kroon, M. J. IJzerman, J. J. Chae, G. J. Lankhorst, and G. Zilvold, "Relation between stimulation characteristics and clinical outcome in studies using electrical stimulation to improve motor control of the upper extremity in stroke," Journal of Rehabilitation Medicine, vol. 37, no. 2, pp. 65-74, 2005.

[3] J. R. D. Kroon, J. H. van der Lee, M. J. Izerman, and G. J. Lankhorst, "Therapeutic electrical stimulation to improve motor control and functional abilities of the upper extremity after stroke: a systematic review," Clin Rehabil, no. 16, pp. 350-360, 2002.

[4] D. N. Rushton, "Functional electrical stimulation and rehabilitation: an hypothesis," Medical Engineering and Physics, vol. 25, 2003.

[5] C. L. Lynch and M. R. Popovic, "Functional electrical stimulation," IEEE Control Systems Magazine, vol. 28, no. 2, pp. 40-50, 2008.

[6] D. Zhang, T. H. Guan, F. Widjaja, and W. T. Ang, "Functional electrical stimulation in rehabilitation engineering: a survey," in Proc. of the 1st int. conv. on rehab. eng. \& assistive tech., 2007, pp. 221-226.

[7] D. Bristow, M. Tharayil, and A. Alleyne, "A survey of iterative learning control," Control Syst Mag, IEEE, vol. 26, no. 3, pp. 96$114,2006$.

[8] C. T. Freeman, A. M. Hughes, J. Burridge, P. Chappell, P. Lewin, and E. Rogers, "Iterative learning control of FES applied to the upper extremity for rehabilitation," Control Engineering Practice, vol. 26 no. 3, pp. 368-381, 2009.

[9] A. M. Hughes, C. T. Freeman, J. Burridge, P. H. Chappell, P. L. Lewin, and E. Rogers, "Feasibility of iterative learning control mediated by functional electrical stimulation for reaching after stroke," Neurorehab. Neural Repair, vol. 23, no. 6, pp. 559-568, 2009.

[10] C. T. Freeman, D. Tong, K. Meadmore, Z. Cai, E. Rogers, A. M. Houghes, and J. H. Burridge, "Phase-lead iterative learning control algorithms for functional electrical stimulation based stroke rehabilitation," Proc. IMechE - Part I: J. Systems \& Control Eng., no. 6, pp. 850-859, 2011.

[11] J. Mehrholz, T. Platz, J. Kugler, and M. Pohl, "Electromechanical and robot-assisted arm training for improving arm function and activities of daily living after stroke," Cochrane Db Syst Rev, vol. 4, 2008

[12] J. N. Ingram, K. P. Körding, I. S. Howard, and D. M. Wolpert, "The statistics of natural hand movements," Experimental Brain Research, vol. 188, no. 2, pp. 223-236, 2008.

[13] E. Theodorou, E. Todorov, and F. J. Valero-Cuevas, "Neuromuscular stochastic optimal control of a tendon driven index finger model," in American Control Conference, 2011.

[14] F. J. Valero-Cuevas, "A mathematical approach to the mechanical capabilities of limbs and fingers," Prog Mot Cont, pp. 619-633, 2009.

[15] J. Landsmeer, "Anatomical and functional investigations on the articulation of the human fingers," Acta Anatomica 24, vol. 2, no. 25, pp. $1-69,1955$.

[16] F. Le, I. Markovsky, C. T. Freeman, and E. Rogers, "Identification of electrically stimulated muscle models of stroke patients," Control Engineering Practice, vol. 18, pp. 396-407, 2010.

[17] C. T. Freeman and Y. Tan, "Iterative learning control with mixed constraints for point-to-point tracking," IEEE Transactions on Control System Technology, vol. PP, no. 99, pp. 1-13, 2012. 